\title{
Polimorfismo de tallas en las cercarias de una cepa venezolana de Schistosoma mansoni
}

\author{
Polymorphism of size in the cercariae of one venezuelan \\ strain of Schistosoma mansoni
}

Luz A. Pino1, Liboria Matinella2 y Gustavo Morales1

\begin{abstract}
Resumen Mediante la impregnación con nitrato de plata, de un lote de 47 cercarias de la cepa C5 de Schistosoma mansoni (Venezuela), se evidenció un polimorfismo de tallas. El morfo estaría conformado por las cercarias mas grandes (entre 152,9 y 218 $\mu \mathrm{m}$ ), este grupo siempre presente en la población de cercarias en menor porcentaje $(29,8 \%)$. El grupo constituido por las cercarias de talla normal, menores de 152,8 (70,2\%). La comparación entre el largo de ambos lotes de cercarias resultó con diferencias estadísticamente significativas $(t=5,5 ; P<0,001)$. Para el total de las cercarias estudiadas, el largo promedio del cuerpo cercariano resultó de 143,9um y el ancho de 68,6um. Se detectó una correlación positiva y significativa entre el ancho y el largo de las cercarias de I a cepa objeto de estudio $(r=0,83 ; P<0,001)$.
\end{abstract}

Palabras-claves: Schistosoma mansoni. Cercaria. Polimorfismo. Talla.

\begin{abstract}
By mean of the silver impregnation of a lot of 47 cercariae belonging to C5 venezuelan Schistosoma mansoni strain, a polymorphism of size was evidenced. The morph was conformed by the greatest cercariae (between $152.9 \mu \mathrm{m}$ to $218.4 \mu \mathrm{m}$ ). This group was ever present in the population of cercariae in less percentage (29.8\%). The length of the normal cercariae was comprised between $119 \mu \mathrm{m}$ to $152 \mu \mathrm{m}$. This group was the dominant in the population: $70.2 \%$. The comparison between the length of both lots of cercariae resulted with significative statistical differences $(t=5.5 ; P<0.001)$. For the total studied cercariae the average of the length was $X=143.9 \mu \mathrm{m}$ and for the wide $X=68.6 \mu \mathrm{m}$. It was found a positive significative correlation between the wide and the length of the cercariae $r=0.83(P<0.001)$.
\end{abstract}

Key-words: Schistosoma mansoni. Cercariae. Polymorphism. Size.

Desde el aislamiento de la cepa C5 de Schistosoma mansoni se ha observado en las múltiples manipulaciones de las cercarias al microscopio para el mantenimiento del ciclo biológico en el laboratorio, una notoria diferencia de tallas, lo cual también fue detectado a nivel del miracidio. Al realizar la impregnación argéntica de un lote de dichas cercarias, con el objetivo de estudiar la quetotaxia10 y dada la facilidad que representaba la disposición de un lote cercarias en montaje semipermanente en goma de cloral, se decidió realizar las mediciones para efectuar un estudio morfométrico de las cercarias de la presente cepa, lo cual constituye el objetivo de este trabajo.

\footnotetext{
1. Laboratorio de Parasitología, Instituto de Investigaciones Veterinarias, Centro Nacional de Investigaciones Agropecuarias, Fondo Nacional de Investigaciones Agropecuarias; 2. Laboratorio Malacológico, División de Endemias Rurales, Dirección de Malariología y Saneamiento Ambiental.

Trabajo parcialmente financiado por Fundacite Aragua (Proyecto DL-SA0036).

Dirección para correspondencia: Dra Luz A. Pino de Morales. Lab. de Parasitología/CENIAP/FONAIAP. Av. Las Delicias, Maracay, Edo. Aragua, Venezuela. Fax: 043 414153; 043414286.

E-mail: gamc@cantv.net

Recibido para publicação em 24/8/98.
} 


\section{MATERIAL Y METODOS}

Material biologico. La cepa C5 de Schistosoma mansoni fue aislada en el Laboratorio Malacológico de la Dirección de Malariología y Saneamiento Ambiental, Maracay, Estado Aragua, Venezuela, a partir de los huevos presentes en las heces de un paciente de la ciudad de San Mateo, Estado Aragua y es mantenida en el mencionado laboratorio desde 1989, en pasajes sucesivos por caracoles Biomphalaria glabrata de la cepa Cagua, Estado Aragua y ratones blancos.

Metodos parasitologicos. Impregnación con nitrato de plata de las cercarias de S. Mansoni. Las cercarias recién emitidas fueron impregnadas en nitrato de plata al $2 \%$, según la técnica de Combes et al 3 , en la cual se establece el montaje semipermanente de las cercarias impregnadas, en goma de cloral de Faure. La finalidad de dicha metodología es realizar el estudio del número y patrón de disposición de las papilas argirófilas sensoriales en la superficie de las cercarias (quetotaxia), el cual tiene valor sistemático10. El material preparado para esos fines, se obtuvo a partir de la exposición a la luz de 5 caracoles infectados y sirvió para efectuar el presente estudio morfométrico.

Se procesó un total de 47 cercarias, las cuales fueron dibujadas mediante el uso de un microscopio binocular provisto de tubo de dibujo, para luego aplicarles un factor de corrección, obtener las medidas en micras $(\mu \mathrm{m})$.

Análisis de los datos. Las 47 cercarias estudiadas fueron clasificadas en dos subpoblaciones para evaluar las diferencias de tallas existentes en la población: grupo "A" de cercarias normales con tallas comprendidas entre 118,0 y $152,8 \mu \mathrm{m}$ y grupo "B" de cercarias grandes, con tallas mayores de $152,8 \mu \mathrm{m}$, el cual es el valor obtenido de $Q_{3}$, es decir del tercero cuartil superior. Se calculó el coeficiente de correlación de Pearson entre el largo y el ancho de las cercarias y la prueba de "t" de Student para comparar las cercarias pequeñas con las grandes 13 .

\section{RESULTADOS}

La foto tomada al microscopio, de las cercarias de la cepa C5 de Schistosoma mansoni, refleja la diferencia de tallas que la caracteriza (Figura 1).

Las estadísticas descriptivas de las tallas de las cercarias de la cepa C5 pueden ser vistas en la Tabla 1.

Las cercarias se dividieron en dos lotes de acuerdo a la talla: A: cercarias normales, de medidas comprendidas entre 118,0 y $152,9 \mu \mathrm{m}$, $\mathrm{X}=135,8 \mu \mathrm{m} ; \mathrm{s}=9,5 ; \mathrm{n}=33$ y $\mathrm{B}$ : cercarias grandes, mayores de $152,9 \mu \mathrm{m}, \mathrm{X}=162,9 \mu \mathrm{m}$; $\mathrm{s}=17,6 ; \mathrm{n}=14$.

En la Tabla 2, se observan las estadísticas descriptivas del largo de las cercarias de la cepa C5, clasificadas en dos lotes de tallas $(<152,8$ y $>152,9 \mu \mathrm{m})$ y la comparación entre ambos grupos, la cual arrojó diferencias estadísticamente significativas $(P<0,001)$. Asimismo, se evidenció la existencia de una correlación positiva y significativa $(r=0,83$; $P<0,001)$ entre el largo y el ancho de las cercarias de la cepa estudiada.

Tabla 1 - Estadísticas descriptivas del largo y del ancho del cuerpo de las cercarias de la cepa C5 de Schistosoma mansoni.

\begin{tabular}{|c|c|c|c|c|c|c|c|}
\hline Variable & $\mathrm{n}$ & $X$ & $\mathrm{~s}$ & Mín & Máx & $Q_{1}$ & $\mathrm{Q}_{3}$ \\
\hline Largo & 47 & 143,9 & 17,5 & 119,8 & 218,4 & 130,7 & 152,9 \\
\hline Ancho & 47 & 68,6 & 9,8 & 51,3 & 91,0 & 60,3 & 77,0 \\
\hline
\end{tabular}

$\mathrm{n}$ = número de cercarias estudiadas; $\mathrm{X}$ = media aritmética; $\mathrm{s}$ = desviación estándar; Mín = valor mínimo observado; Máx = valor máximo observado; $Q_{1}=$ primer cuartil; $Q_{3}=$ tercer cuartil.

\begin{tabular}{|c|c|c|c|c|c|c|}
\hline Grupo de cercarias & $\mathrm{n}$ & $X$ & s & Mín & Máx & $P$ \\
\hline \multirow[t]{2}{*}{$\bar{A}$} & 33 & 135,8 & 9,5 & 119,8 & 151,1 & \\
\hline & & & & & & 0.001 \\
\hline B & 14 & 162,9 & 17,4 & 152,6 & 218,4 & \\
\hline
\end{tabular}




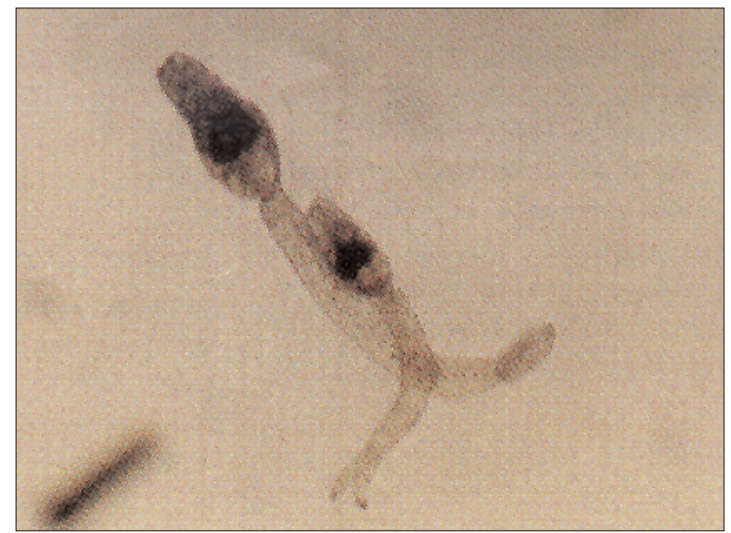

Figura 1 - Microfotografía de las cercarias de la cepa C5 de Schistosoma mansoni.

\section{DISCUSION}

El polimorfismo ha sido definido como la coexistencia en una misma localidad o localización de dos o mas formas discontinuas de una especie en proporciones tales que la mas escasa entre ellas no puede mantenerse solamente por mutación recurrente 4 .

S. Mansoni es una especie polimórfica, hecho que ha sido bastante estudiado sobre todo a nivel del sistema sensorial superficial de las cercarias, cuyas características han sido utilizadas para establecer diferenciaciones infraespecíficas y caracterizaciones de cepas. En efecto, BayssadeDufour utilizó este tipo de criterios para diferenciar cepas del parásito que hacían pasajes por hospedadores vertebrados murinos o humanos 1 , asi como para diferenciar aquellas que utilizaban como hospedador intermediario a Biomphalaria pfeifferi o a B. glabrata, es decir para discriminar entre cepas de S. mansoni africanas o americanas ${ }^{2}$. Mientras que esa metodología también ha sido de utilidad para diferenciar cepas S. Mansoni del Brasil que utilizaban como hospedador intermediario a $B$. tenagophila 7 , a B. glabrata o a B. straminea9.

En S. Mansoni, también fue evidenciado otro fenómeno de polimorfismo, en este caso un dimorfismo sexual, a nivel del sistema sensorial superficial de las cercarias 812 impregnadas con nitrato de plata.

A diferencia de estos casos en los cuales se utilizó el polimorfismo para diferenciaciones infraespecíficas, en el presente trabajo se estudia un caso de polimorfismo de tallas, al interior de un cepa.
Pino6, en cercarias de la misma cepa y sometidas a la misma técnica de impregnación, pero montadas en dos líquidos diferentes: goma de cloral de Faure y Berlesse, ambos preparados en base a goma arábiga, señala la existencia de diferencias estadísticamente significativas entre los tamaños de los dos lotes de cercarias. Por lo que se considera de suma importancia en estudios morfométricos, suministrar información sobre el procesamiento al cual se sometió el material biológico en estudio, de manera de que los resultados obtenidos puedan ser comparables.

La marcada diferencia de tallas observada en la cepa C5 no se ha detectado en otras cepas venezolanas, ni hemos encontrado reportes al respecto en la bibliografía. Para otras cepas venezolanas, a las cuales le hemos estudiado la quetotaxia10 11 y que por lo tanto han sido sometidas al mismo tratamiento, tenemos las siguientes medidas: Cepa "JR" X $=144,9 \mu \mathrm{m}$; Valor mínimo (Vmí) =136,1 $\mu$ m y Valor máximo $($ Vmá $)=174,7 \mu \mathrm{m}$. Cepa Caraballeda $\mathrm{X}=$ $151,2 \mu \mathrm{m}$, Vmí $=119,4$, Vmá $=173,6 \mu \mathrm{m}$. No se encontraron nunca valores extremos máximos, comparables a los observados en la cepa C5.

En el caso de las cercarias de la cepa C5, se considera que existe un fenómeno de polimorfismo, pues el lote de cercarias grandes siempre está presente y en menor porcentaje $(29,8 \%)$ por lo que se le puede considerar el morfo minoritario o alternativo, siendo las cercarias clasificadas como pequeñas, las dominantes $(70,2 \%)$. 


\section{REFERENCIAS BIBLIOGRAFICAS}

1. Bayssade-Dufour Ch. Variation chétotaxique chez les cercaires de Schistosoma mansoni agent de la bilharziose intestinale, en corrélation avec l'hôte vertebré de ce parasite. Cahiers de la Real Academie de Sciences, série D 285:1511-1513, 1977a.

2. Bayssade-Dufour Ch. Differences au niveau des sencilles entre les cercaires africaines et américaines de Schistosoma mansoni agent de la bilharziose intestinale humaine. Cahiers de la Real Academie de Sciences, série D 284:191-193, 1977b.

3. Combes C, Bayssade-Dufour Ch, Cassone J. Sur l'impregnation et le montage des cercaires pour l'étude chétotaxique. Annales de Parasitologie Humaine et Comparée 5:399-400, 1976.

4. Ford E. Génétique écologique. Gauthiers Villars, Paris, 1972.

5. Pifano F. La Schistosomiasis mansoni en Venezuela y sus problemas. Archivos venezolanos de Medicina Tropical y Parasitología Médica 5:195-224, 1973.

6. Pino LA. El uso de la quetotaxia cercariana para la diferenciación de sexos y caracterización de cepas de Schistosoma mansoni. Con especial referencia a la interpretación estadística. Trabajo de Ascenso Prof. Titular, Universidad de los Andes, Mérida, Venezuela.

7. Pino LA, Freire N, Morales G. Quetotaxia cercariana de Schistosoma mansoni proveniente de Biomphalaria tenagophila naturalmente infectadas. Acta Científica Venezolana 37:56-59, 1986.

8. Pino LA, Morales G. Análisis discriminante y diferenciación de sexos de Schistosoma mansoni a nivel del estadío cercaria. Revista Ibérica de Parasitología 50:233-240, 1990

9. Pino LA, Morales G, Freire N. Comparación de la quetotaxia entre dos grupos de cercarias de una cepa de origen humano de Schistosoma mansoni luego de un primer pasaje por dos especies de moluscos congenéricas. Acta Científica Venezolana 37:60-62, 1986b.

10. Pino LA, Morales G, Matinella L. Quetotaxia cercariana de dos cepas venezolanas de Schistosoma mansoni. Boletín Chileno de Parasitología 52:61-66, 1997.

11. Pino LA, Morales G, Morales LA. Quetotaxia cercariana de la cepa Caraballeda de Schistosoma mansoni. Parasitología al Día 22:11-15, 1998.

12. Pino LA, Morales G, Noya B Alarcon de, Noya O. La quetotaxia cercariana en la diferenciación de sexos de Schistosoma mansoni. Memórias do Instituto Oswaldo Cruz, 83:367-374, 1988.

13. Sokal R, Rohlf F. Biometría. H. Blume Ediciones, Madrid, España, 1979. 